\title{
Cranial Meningocele
}

National Cancer Institute

\section{Source}

National Cancer Institute. Cranial Meningocele. NCI Thesaurus. Code C124517.

A congenital abnormality in which the meninges protrude through a defect in the cranium. 